\title{
MEASUREMENTS OF FOREST CHANGE USING P-BAND SAR BACKSCATTER
}

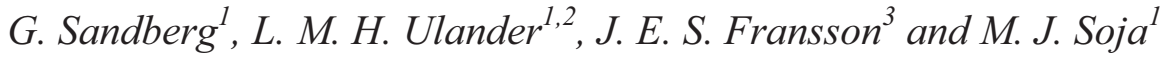 \\ ${ }^{1}$ Chalmers University of Technology, ${ }^{2}$ Swedish Defense Research Agency \\ ${ }^{3}$ Swedish University of Agricultural Sciences
}

\begin{abstract}
Using data from the recent BioSAR 2007 and BioSAR 2010 campaigns, it has for the first time been possible to measure forest biomass change using P-band SAR data. Regression models based on backscatter change have been developed using reference data derived from high density laser scanning data. The models were evaluated for six areas with detailed in-situ measurements, for which the maximum biomass loss and growth was $30 \%$ and $20 \%$, respectively. For the best model the coefficients of determination was 55$89 \%$. This result suggests that not only clear cuts but also forest growth and thinning can be measured using P-band SAR backscatter.
\end{abstract}

Index Terms - SAR, P-band, biomass change

\section{INTRODUCTION}

A very promising method for monitoring of forest biomass change is by means of low frequency Synthetic Aperture Radar (SAR). Strong sensitivity between L- and P-band backscatter and biomass have been found in numerous studies (e.g. [6]), with highest sensitivity for P-band. However, the literature on measurements of forest change is scarce. Exceptions for L-band include [1] and [3], but to the authors knowledge no studies using experimental P-band data for measurement of forest change have been made.

In this paper we present the first measurements of biomass change based on P-band SAR data. The analysis is based on data from the recent BioSAR 2007 and BioSAR 2010 campaigns, launched in support of the proposed ESA Earth explorer mission BIOMASS. Regression models based on change in backscatter are developed, as well as a method to correct for changes in backscatter caused by changes in moisture and radiometric calibration uncertainty.

The results suggest that not only clear cuts but also forest growth and thinning can be measured using P-band backscatter. An extended analysis is presented in [5].

\section{DATA DESCRIPTION}

The data used in this paper were collected within two experimental campaigns, BioSAR 2007 and BioSAR 2010, both funded by the European Space Agency (ESA). Both campaigns were conducted in Remningstorp in southern Sweden. Complete campaign descriptions can be found in [2] and [4] for BioSAR 2007 and in [7] for BioSAR 2010. A brief summary of the data is given below.

\subsection{In-situ data}

Two different types of in-situ measurements have been used in this paper. The first is a set of seven $80 \mathrm{~m}$ by $80 \mathrm{~m}$ squares. For these squares measurements were made on all trees with a diameter greater than $5 \mathrm{~cm}$ in both 2006-2007 and 2010-2011. A summary of plot level data for six of the seven squares is given in Table 1 . The seventh square was not included in the analysis since it was not covered by all SAR images.

The other type of in-situ data are systematic grids of measurement plots. In 2004-2005 measurements were made for a dense grid covering the central part of Remningstorp. In 2010, a sparser grid covering the full test site was used. For the latter grid, both biomass and stem volume were estimated for each plot, while for the former only stem volume estimates were made. Due to a storm event between 2004 and 2007, Remningstorp was affected by storm damage. After the storm event, wind felled trees in the central part of the site were located and their positions were measured using GPS.

\subsection{Biomass maps}

High density laser scanning data were collected both 2007 and 2010 using the helicopter borne system TopEye. In 2007 data were collected from the central part of the site while in 2010 the full site was covered. The laser scanning

Table 1: Plot level data for seven $80 \mathrm{~m}$ by $80 \mathrm{~m}$ squares. Measurements were made for all trees with a diameter at breast height larger than $5 \mathrm{~cm}$.

\begin{tabular}{lllll}
\multirow{2}{*}{ Plot Id } & \multicolumn{2}{c}{ Number of trees } & \multicolumn{2}{c}{ Biomass [t/ha] } \\
\cline { 2 - 5 } & $\mathbf{2 0 0 7}$ & $\mathbf{2 0 1 0}$ & $\mathbf{2 0 0 7}$ & $\mathbf{2 0 1 0}$ \\
\hline 1 & 278 & 265 & 202 & 201 \\
5 & 552 & 176 & 160 & 109 \\
10 & 400 & 393 & 150 & 174 \\
14 & 346 & 334 & 52 & 64 \\
17 & 377 & 366 & 142 & 174 \\
18 & 317 & 312 & 246 & 285
\end{tabular}


data were used together with the systematic plot grid measurements and species stratifications based on aerial photography to derive biomass maps with a $10 \mathrm{~m}$ by $10 \mathrm{~m}$ cell size. In 2007 a stem volume map was first derived, which was then converted to biomass using species dependent biomass expansion factors [4]. This indirect approach was needed since only stem volume estimates were available for the systematic plot grid from 2004-2005. In 2010 an intermediate stem volume map was not used.

The biomass the maps were filtered and downsampled to obtain a resolution of $50 \mathrm{~m}$ by $50 \mathrm{~m}$. Areas overlapping with the $80 \mathrm{~m}$ by $80 \mathrm{~m}$ squares were removed. Biomass change maps were then obtained through differentiation of the maps. This approach gives higher errors than direct change estimates [1], but a direct approach could not be used due to a lack of in-situ reference data suitable for model training.

\subsection{SAR data}

In BioSAR 2007 L- and P-band SAR data were collected using the German system ESAR, owned and operated by the German Aerospace Center (DLR). Images were acquired on three occasions: 9 March 2007, 31 March \& 2 April 2007, and 2 May 2007. Two flight tracks with headings $200^{\circ}$ and $179^{\circ}$, respectively, were used. For the latter track steep incidence angles were used for the central part of the site. On 23 September 2010 the measurements of BioSAR 2007 werer repeated in the BioSAR 2010 campaign, but his time using ONERAS SAR system SETHI. L- and P-band data were acquired with image geometries matching those of BioSAR 2007. A third flight track was also flown to enable studies of topographic variability.

The calibrated images were corrected for variations due to incidence angle according to $\gamma^{0}=\sigma^{0} / \cos \left(\theta_{\mathrm{i}}\right)$, where $\theta_{\mathrm{i}}$ is the local incidence angle and $\sigma^{0}$ is the average radar cross section per unit ground area. For convenience, $\gamma^{0}$ will hereafter be referred to as backscatter. Backscatter maps matching the geometry of the biomass maps were created by filtering and re-sampling of the full resolution backscatter images.

In order to reduce effects of e.g. incident angle and topography, the analysis was restricted to pairwise image comparisons for images with the same image geometry. When repeat measurements (i.e. images from the same date and flight track) were available, only one of these was chosen for analysis.

\section{METHOD}

The biomass estimation method proposed in this paper consists of two parts. First backscatter offsets caused by changes in environmental conditions and calibration uncertainties are corrected. Then the offset-corrected backscatter is used to build regression models for biomass change estimation.

\subsection{Offset correction method}

Variations in backscatter are not only caused by changes in biomass. Environmental effects such as soil and stem moisture, as well as calibration uncertainty, also influence the backscatter. Thus, it is unlikely that a zero change in biomass directly corresponds to a zero change in backscatter. Indeed, for the data used in this paper the backscatter change corresponding to unchanged biomass (i.e. the backscatter offset) is up to $2.7 \mathrm{~dB}$ for $\mathrm{HH}$ and $\mathrm{VV}$ and up to $2 \mathrm{~dB}$ for $\mathrm{HV}$.

However, it has been observed the HH-VV backscatter ratio is less susceptible to changes in moisture than any of the linearly polarized backscatter combinations, at least in boreal forest. Moreover, the ratio will be less sensitive to calibration offsets between two images as long as the offset is similar for $\mathrm{HH}$ and VV. Furthermore, for the data used in this paper the $\mathrm{HH}-\mathrm{VV}$ ratio is sensitive to change in biomass, albeit weakly so. Thus, areas with near-zero change in $\mathrm{HH}-\mathrm{VV}$ ratio are areas for which the change in biomass is low, which leads us to propose the following offset correction method:

1) Select areas for which the absolute change in $\mathrm{HH}-\mathrm{VV}$ ratio, on $\mathrm{dB}$ scale, is smaller than a threshold value.

2) Calculate the mean backscatter level for the selected areas for each polarization and both images in an image pair.

3) For each polarization, force the mean backscatter level for the selected areas in both images to be equal by applying a correction factor to each image.

\subsection{Model selection}

After offset correction, the most suitable choice of estimation function needs to be determined. A general description of the biomass change estimation functions investigated in this paper is given by (1), where $y$ is biomass change measured as a difference on log (natural logarithm), square root or linear scale. $\mathrm{x}_{\mathrm{PQ}}$ is backscatter change, measured as a difference in amplitude, power or decibel $(\mathrm{dB})$, for polarization $\mathrm{PQ}$, and $b_{i}, \mathrm{i}=0,1, \ldots, 8$, are regression coefficients.

$$
\begin{gathered}
y=b_{0}+b_{1} x_{H V}+b_{2} x_{H V}^{2}+b_{3} x_{H H}++b_{4} x_{H H}^{2} \\
+b_{5} x_{V V}+b_{6} x_{V V}^{2}+ \\
+b_{7}\left(x_{H H}-x_{V V}\right)+b_{8}\left(x_{H H}-x_{V V}\right)^{2}
\end{gathered}
$$

For each choice of $y$ and $x$ the best choice of regression parameters was determined. This was done through a separation of data into disjoint training and validation sets. 500 random selections of training and validation areas were made for each choice of $y$. For each selection of training areas and each image pair, regression coefficients were estimated for all sub-models of (1) with up to 5 non-zero regression coefficients. Models trained on one image pair were then applied to all image pairs (including the one used for training), giving 36 combination of training-evaluation image pairs. For each such pair the mean squared difference 
(msd) and bias were calculated for the selected validation areas. In addition, bias and mean squared error (mse) was calculated for the $80 \mathrm{~m}$ by $80 \mathrm{~m}$ squares. The usage of the notation msd instead of mse for the biomass change estimates based on laser scanning data reflects the fact that these estimates may contain large errors.

\section{RESULTS}

The results are organized as follows. First, an evaluation of the offset correction method is presented. Then the results of the model selection are presented, and the selected models are evaluated using the $80 \mathrm{~m}$ by $80 \mathrm{~m}$ squares. Finally, the selected models are used to create biomass maps for the central part of Remningstorp, which are compared to maps based on laser scanning data.

\subsection{Offset correction method}

The offset correction method was evaluated by estimating offsets before and after offset correction. In the evaluation the offsets were estimated as mean backscatter differences for areas with little change in biomass. Such areas were selected based on the biomass maps based on laser data. Different thresholds for the HH-VV ratio were tested: 0.5 , 1.0 and $2.0 \mathrm{~dB}$. Without correction, the offset is up to 2.7 $\mathrm{dB}$, which is reduced to a negligible $0.2 \mathrm{~dB}$ using a threshold of $2.0 \mathrm{~dB}$. The performance is only weakly sensitive to the choice of threshold; the residual offset is at most $0.6 \mathrm{~dB}$ for all listed threshold values.

\subsection{Model selection}

Model performance was evaluated by studying mean square differences and bias for the $50 \mathrm{~m}$ by $50 \mathrm{~m}$ cells in the biomass change map, grouped by biomass change class. Mean squared errors for the $80 \mathrm{~m}$ by $80 \mathrm{~m}$ squares were also studied. Based on these data, no unambiguous selection of a "best" prediction model could be made, since many models show similar performance. Nonetheless, some general conclusion can be made. The single most informative predictor is change in HV backscatter. Adding additional predictors give lower estimation errors, but the decrease in error obtained by including more than two predictors does not justify the increased model complexity.

For the $\log$ and square root models the lowest errors were obtained by measuring backscatter change on decibel (dB) scale. For the linear model, backscatter change measured on $\mathrm{dB}$ or amplitude scale yields similar results, but the amplitude model gave lower estimation errors for the $80 \mathrm{~m}$ by $80 \mathrm{~m}$ squares as well as smaller estimation bias for areas with high growth.

One model for each biomass change scale was selected for further analysis of estimation performance. For the linear and square root models, $\mathrm{HV}$ and $\mathrm{VV}$ were used as predictors. For the log model the choice of a second predictor, to complement $\mathrm{HV}$, was less straightforward. Models with a square term ranked among the models with lowest errors, which are explained by a slightly non-linear relation between biomass change and backscatter change. However, the model with $\mathrm{HV}$ and $\mathrm{HH}$ and no square terms gave was selected since it gave lower estimation bias for high loss and high growth areas.

\subsection{Model evaluation}

The selected models were applied on the six $80 \mathrm{~m}$ by $80 \mathrm{~m}$ squares with in-situ measurements for each image pair. For these squares the maximum biomass loss was about $30 \%$ (due to thinning) and the maximum biomass growth was about $20 \%$.

Coefficients of determination $\left(\mathrm{R}^{2}\right)$ was calculated for every image pair and biomass change scale for the estimates based on P-band backscatter, and are shown in Table $2 . \mathrm{R}^{2}$ based on laser scanning data are also shown in the table. $\mathrm{R}^{2}$ was calculated using the formula $\mathrm{R}^{2}=1-\sum\left(\widehat{y_{k}}-y_{k}\right)^{2}$ / $\sum\left(y_{k}-\bar{y}\right)^{2}$, where $\widehat{y_{k}}$ are change estimates, $y_{k}$ are reference change measurements and the bar denotes sample mean. Best results were obtained using the linear model, with a best case $\mathrm{R}^{2}$ of $89 \%$. The $\mathrm{R}^{2}$ for the log model varies greatly across images, and in the worst case the estimation errors are larger than for a constant model with correct mean (negative $\mathrm{R}^{2}$ ). A somewhat surprising result is that the radar gives higher $\mathrm{R}^{2}$ than the laser in all but one case. Although partially explained by a non-optimum use of laser data, this result indicates the strong potential for low error biomass change estimation using P-band SAR.

\subsection{Biomass maps}

Biomass change maps created using the log and linear models are shown in Figure 1 for one image pair (see figure caption). Other image pairs give similar results, as does the square root model. There is a generally good agreement between the change maps based on laser scanning data and those based on P-band data.

Table 2: Coefficient of determination $\left(R^{2}\right)$ in percent for the $80 \mathrm{~m}$ by $80 \mathrm{~m}$ squares for each image pair. The selected models for each biomass change scale are described in section 4.2. The roman numbers indicate the image acquisition occasion for ESAR: 9 March 2007 (I), 31 March 2007 \& 2 April 2007 (II), and 2 May 2007 (III). The negative $\mathrm{R}^{2}$ indicates that the model performs worse than a constant model with correct mean.

\begin{tabular}{|c|c|c|c|c|c|c|c|}
\hline \multirow{2}{*}{$\begin{array}{l}\text { Biomass } \\
\text { change } \\
\text { scale }\end{array}$} & \multicolumn{3}{|c|}{$\begin{array}{l}\text { Flight heading } \\
200^{\circ}\end{array}$} & \multicolumn{3}{|c|}{$\begin{array}{l}\text { Flight heading } \\
179^{\circ}\end{array}$} & \multirow{2}{*}{$\begin{array}{l}\text { Laser } \\
\text { scanning } \\
\text { data }\end{array}$} \\
\hline & I & II & III & I & II & III & \\
\hline $\log$ & 28 & 69 & 44 & 28 & 48 & -19 & 9 \\
\hline $\begin{array}{l}\text { Square } \\
\text { root }\end{array}$ & 68 & 75 & 74 & 60 & 75 & 68 & 28 \\
\hline Linear & 78 & 88 & 89 & 55 & 80 & 64 & 34 \\
\hline
\end{tabular}



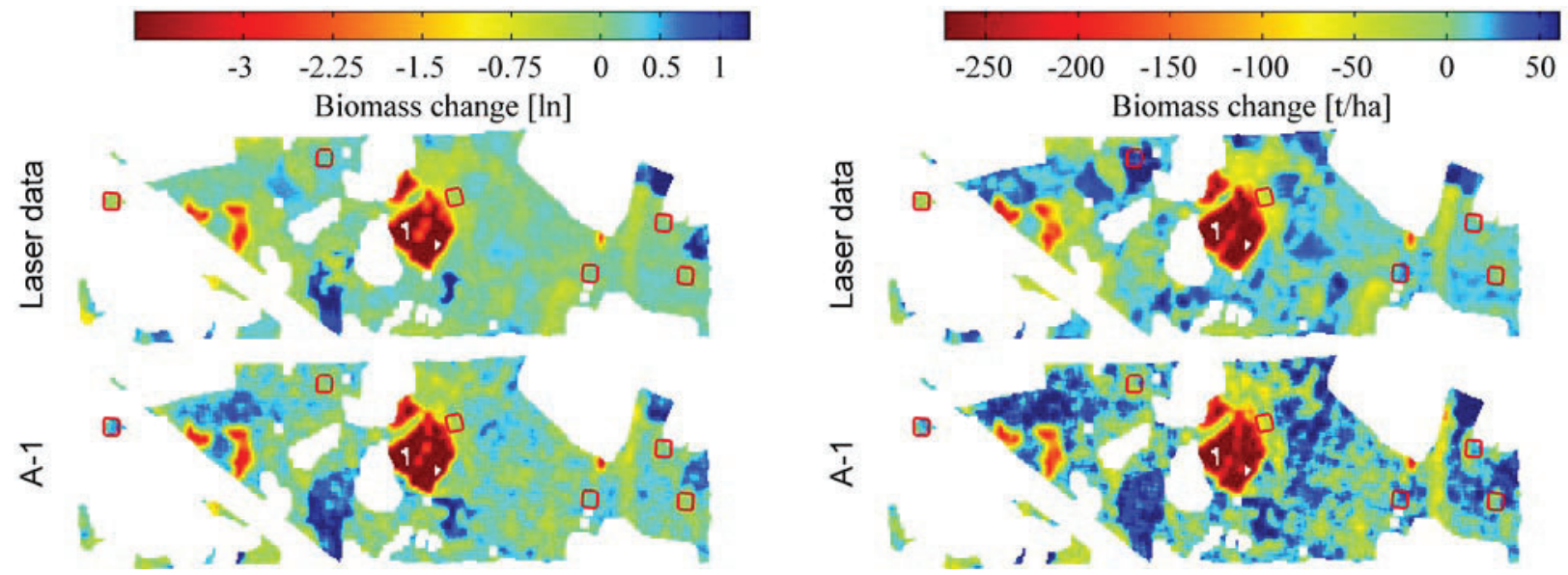

Figure 1: Comparison of biomass change maps based on laser scanning data and P-band backscatter on log (left) and linear scale (right). The maps have been rotated 90 degrees clockwise. The two maps for each change scale show biomass change estimates based on laser data (top) and P-band backscatter from heading $200^{\circ}$, acquired on 9 March 2007 and 23 September 2010 (bottom). The contours of the $80 \mathrm{~m}$ by $80 \mathrm{~m}$ squares with in-situ measurements are overlaid in red. The Plot Ids for the squares are, from left to right (i.e. ascending northing): 14 , $1,5,18,17$ and 10. It should be noted that the reference map based on laser data does not represent ground truth. In areas where the change maps based on laser and P-band data disagree, it is presently unsettled which of the two maps that gives the best estimate.

Both loss regions and growth regions are clearly seen in the change maps. Small scale features such as variability within the large central clear cut are also seen. It should be stressed that the change maps based on laser data contain errors. In areas where the change maps based on laser and P-band data disagree, it is presently unsettled which of the two maps that gives the best estimate.

The interpretation of the biomass maps is facilitated by the overlaid $80 \mathrm{~m}$ by $80 \mathrm{~m}$ squares. For example, the square in the center of the map was thinned between 2007 and 2010. Both the laser and the radar correctly indicate biomass loss, and it can be seen that the square is part of a larger thinned area.

\section{DISCUSSION AND CONCLUSIONS}

For the first time, experimental P-band SAR data have been used to estimate changes in forest biomass. Using reference data derived from laser scanning data, regression models for estimation of biomass change based on P-band backscatter were developed. The models were evaluated for six areas with detailed in-situ measurements, for which the maximum biomass loss and growth was $30 \%$ and $20 \%$, respectively. For the best model the coefficients of determination was 55$89 \%$. This result suggests that not only clear cuts but also forest growth and thinning can be measured using P-band SAR backscatter.

A method for correction of backscatter change caused by environmental effect (e.g. moisture changes) and radiometric calibration uncertainty was also developed. The method is based on the HH-VV backscatter ratio. Without correction, a backscatter change of at most $2.7 \mathrm{~dB}$ was measured for areas with little change in biomass. The correction method was able to reduce this offset to a negligible $0.2 \mathrm{~dB}$.
The results in this paper indicates a strong potential for biomass change estimation using P-band SAR backscatter. However, it should be stressed that the results are based on a single test site for a single change period. Although we believe that similar results can be produced in other areas, it is apparent that more data like those from the successful BioSAR 2007 and BioSAR 2010 campaigns are needed to confirm this belief.

\section{REFERENCES}

[1] H. Balzter, L. Skinner, A. Luckman, and R. Brooke, "Estimation of tree growth in a conifer plantation over 19 years from multi-satellite L-band sar," Remote Sensing of Environment, vol. 84, pp. $184-191,2003$.

[2] I. Hajnsek et al., "BioSAR 2007 technical assistance for the development of airborne SAR and geophysical measurements during the BioSAR 2007 experiment: Final report without synthesis," European Space Agency, Contract no.: 20755/07/NL/CB, 2008.

[3] C. S. Rowland, H. Balzter, T. P. Dawson, A. Luckman, G. Patenaude, and L. Skinner, "Airborne sar monitoring of tree growth in a coniferous plantation," International Journal of Remote Sensing, vol. 29, pp. 3873-3889, 2008.

[4] G. Sandberg, L. Ulander, J. Fransson, J. Holmgren, and T. L. Toan, "L- and P-band backscatter intensity for biomass retrieval in hemiboreal forest," Remote Sensing of Environment, vol. 115, pp. $2874-2886,2011$.

[5] G. Sandberg, L. M. H. Ulander, J. Wallerman, J. E. S. Fransson, and J. Holmgren, "Measurements of biomass change using P-band SAR backscatter", manuscript

[6] T. Le Toan, A. Beaudoin, J. Riom, and D. Guyon, "Relating forest biomass to SAR data," IEEE Transactions on Geoscience and Remote Sensing, vol. 30, pp. 403-411, 1992.

[7] L. M. H. Ulander et al., "BioSAR 2010 Technical Assistance for the Development of Airborne SAR and Geophysical Measurements during the BioSAR 2010 Experiment: Final Report," ESA contract no. 4000102285/10/NL/JA/ef, 2011. 